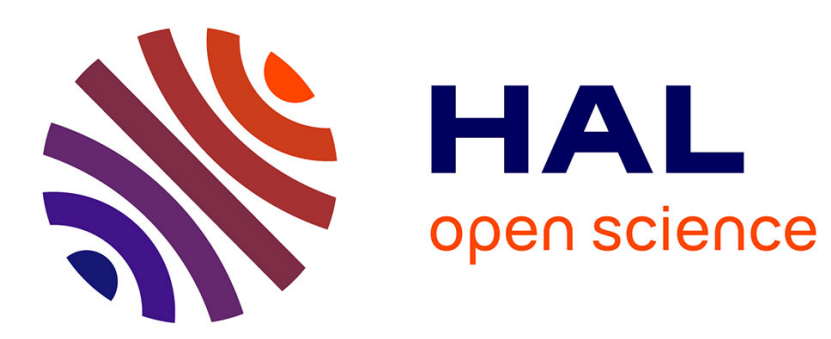

\title{
On Cyclical Hygrothermal Fields in Laminated Plates
}

Marco Gigliotti, Frédéric Jacquemin

\section{To cite this version:}

Marco Gigliotti, Frédéric Jacquemin. On Cyclical Hygrothermal Fields in Laminated Plates. Journal of Composite Materials, 2013, 47, pp.231-242. 10.1177/0021998312439083 . hal-01007143

\section{HAL Id: hal-01007143 https://hal.science/hal-01007143}

Submitted on 10 Mar 2018

HAL is a multi-disciplinary open access archive for the deposit and dissemination of scientific research documents, whether they are published or not. The documents may come from teaching and research institutions in France or abroad, or from public or private research centers.
L'archive ouverte pluridisciplinaire HAL, est destinée au dépôt et à la diffusion de documents scientifiques de niveau recherche, publiés ou non, émanant des établissements d'enseignement et de recherche français ou étrangers, des laboratoires publics ou privés. 


\title{
On cyclical hygrothermal fields in laminated plates*
}

\author{
Marco Gigliotti' and Frédéric Jacquemin ${ }^{2}$
}

\begin{abstract}
This article focuses on the calculation of transient and cyclical hygrothermal fields in laminated composite plates. Water diffusion is simulated by employing Fick's law, while thermal fields are assumed to be uniform: temperature and water concentration are coupled through the coefficient of hygroscopic diffusivity, which follows a classical Arrhenius law. Relative humidity and temperature at the plate boundary vary in a transient/cyclic way, reproducing real complex environmental conditions. Analytical solutions of the cyclical hygrothermal fields in laminated plates can be employed as benchmark for numerical calculations and for evaluating the induced hygrothermal stress, therefore, for structural, design and optimisation purposes.
\end{abstract}

\author{
Keywords \\ Cyclical hygrothermal field, laminated plate
}

\section{Introduction}

Hygrothermal conditioning may represent an important source of ageing and degradation, especially in polymer-based composite laminate structures. Water diffusion may contribute to material properties degradation and leads to the development of anelastic strains of hygroscopic nature, which contribute to the building of internal stress. High temperature may be also responsible for material properties changes and for the development of thermal stresses, leading possibly to damage onset and propagation.

Numerous research studies - both theoretical and experimental - have been carried out on the effect of a hygrothermal environment on the behaviour of laminated composite materials, starting from the pioneering works by Springer et al. ${ }^{1}$ A review paper by Weitsman ${ }^{2}$ cites few hundred papers and the list is not exhaustive. Despite all these important efforts, not much attention has been paid to the real hygrothermal conditions encountered by laminated composite materials during their service and operating life. ${ }^{3}$

'Laboratory' test conditions put usually the samples very far away from the actual environment affecting the structure: the certification authority starts from the principle that often materials subjected to a hygrothermal environment reach a saturated state, in which the residual properties of the material can be comprehensively characterised, and suggests to take into account the properties of the saturated 'wet' state for design. This procedure, which is well established and simple, openly ignores the transient and cyclical phases of the actual conditioning that precede a saturated state, which sometimes is never attained.

A transient/cyclical hygrothermal state may be characterised by strong concentration and material property gradients, varying with time, which may in turn lead to strain/stress gradients and interact with other sources of degradation affecting the durability of the structure in an unpredictable way.

Even from the modelling side, the available tools to handle the problem of laminated plates under hygrothermal conditioning usually reduce to the classical

\footnotetext{
'Département de Physique et Mécanique des Matériaux, Institut Pprime, CNRS, ENSMA, Université de Poitiers, Chasseneuil, France

${ }^{2}$ Institut de Recherche en Génie Civil et Mécanique, Université de Nantes, Boulevard de l'Université, Saint-Nazaire, France

*This article is dedicated to the memory of our master, friend and colleague Prof. Alain Vautrin.

\section{Corresponding author:}

Marco Gigliotti, Département de Physique et Mécanique des Matériaux, Institut Pprime, CNRS, ENSMA, Université de Poitiers, Chasseneuil, France.

Email: marco.gigliotti@ensma.fr
} 
Fick's law with simple boundary conditions, mainly uniform concentration at the structure edges for which analytical solutions are available. ${ }^{4}$

The calculation of the hygrothermal fields for laminated cylinders exposed to complex transient/cyclical environment has been carried out by Jacquemin and Vautrin, ${ }^{5}$ but an analogous formulation for laminated plates has never been presented.

This article is concerned with the case of a laminated plate subjected to cyclical hygrothermal conditions. Temperature and relative humidity $(\mathrm{RH})$ vary in a periodical (transient/cyclical) manner on the external surfaces of the plate, while the values of moisture concentration within the solid are governed by the Fick's law of diffusion, which is formally identical to the Fourier's law for thermal conduction. ${ }^{4,6}$ Analytical solutions of the cyclical hygrothermal fields in laminated plates can be employed as benchmark for numerical calculations; moreover, they can be used for evaluating the induced hygrothermal stress, ${ }^{7-10}$ ), therefore, for structural, design and optimisation purposes.

The present formulation generalises an approach for homogeneous diffusion already presented by Gigliotti et al. ${ }^{10}$ to the case of laminated composite plates, in which different plies have different material properties.

Despite several research studies have been carried out on the interaction between damage and water diffusion, ${ }^{11-13}$ the approach neglects the occurrence/presence of damage and its effect on the diffusion process. For instance, it should be noted that - at temperatures higher than $100^{\circ} \mathrm{C}$ - the absorbed moisture could turn into steam, causing internal damage due to steam blistering: analogous - though different - phenomena may take place at low temperatures (lower than $0^{\circ} \mathrm{C}$ ), since water eventually entrapped within voids of cracks may undergo solidification inducing damage onset and propagation. All these issues are complex and require specific theoretical and experimental dedicated research which is out of the scope of this article. These research topics will be the object of future investigations.

'Analytical model' of a laminated composite plate subjected to hygrothermal fluctuations are presented in the following section. Subsequent section provides a simplified version of the model, valid for 'Homogeneous plates'. Then, 'Application to aircraft plate structures subjected to supersonic flight environments' section presents - for the scope of illustration some results issued from simulations in case of industrial/practical interest. Finally, some 'Conclusion and perspectives' are drawn.

\section{Analytical model}

The equation of unidirectional Fick's diffusion (along the direction $z$, for instance the thickness), for a laminated plate with thickness $e$ and made by $n$ laminae/layers with thickness $e_{i}$ and for $0<z_{i}<e_{i}$ reads

$$
\begin{aligned}
& \frac{\partial c_{i}\left(z_{i}, t\right)}{\partial t}=D_{i}(t) \frac{\partial^{2} c_{i}\left(z_{i}, t\right)}{\partial z_{i}^{2}} \\
& \forall 0<z_{i}<e_{i}, t>0 \\
& \forall i, i=1, \ldots, n
\end{aligned}
$$

where $c_{i}$ is the water concentration within the $i$ th lamina. For an environment in which hygrothermal conditions vary in a periodic way, the following boundary and interface conditions may be written

$$
\begin{aligned}
c_{i}\left(z_{i}, 0\right) & =0 \\
c_{i}\left(e_{i}, t\right) & =\alpha_{i, i+1} c_{i+1}(0, t), \quad \forall i, i=1 \ldots n-1 \\
D_{i}(t) \frac{\partial c_{i}\left(e_{i}, t\right)}{\partial z_{i}} & =D_{i+1}(t) \frac{\partial c_{i+1}(0, t)}{\partial z_{i+1}} \forall i, i=1 \ldots n-1 \\
c_{1}(0, t) & =c_{a}(t) \\
c_{n}\left(e_{n}, t\right) & =c_{b}(t)
\end{aligned}
$$

where $c_{a}(t)$ and $c_{b}(t)$ are the surface water concentrations, both depending on time, and $D_{i}(t)$ the diffusion coefficient within the $i$ th layer. In equations (1) and (2), boundary conditions and diffusion coefficients depend on time: in particular, the layer diffusion coefficient depends on temperature through the Arrhenius law

$$
D_{i}(t)=A_{i} \exp \left(\frac{B_{i}}{T(t)}\right)
$$

where $A_{i}$ and $B_{i}$ are material constants. Temperature is supposed to be uniform along the laminated plate, that is, thermal transport phenomena are supposed to be much faster than water concentration diffusion phenomena, which is physically reasonable in most practical situations. $\alpha_{i, i+1}$ is a constant which expresses the ratio between the saturated concentration of the $i$ th layer and that of the adjacent $(i+1)$ th layer.

Usually, at layer interfaces, continuity of the water concentration is imposed. A more correct condition consists in imposing continuity of the chemical potential: the use of a proper constant coefficient $\alpha_{i, i+1}$ (not necessarily equal to 1) allows for this condition to be treated.

Let us now consider the case in which hygrothermal conditions are cyclical, that is, temperature and water concentration boundary conditions vary with time periodically with period $\tau$.

In order to analytically solve the problem, a change of variables is needed: the initial problem equations (1) and (2) can be then substituted by an analogous problem in which the boundary conditions and the diffusion 
coefficient are constant. Let us consider the following change of variables

$$
u_{i}(t)=\frac{\int_{0}^{t} D_{i}(q) \mathrm{d} q}{\int_{0}^{\tau} D_{i}(q) \mathrm{d} q}=\frac{D_{i}(t)}{\Delta_{i}(\tau)} \Rightarrow \mathrm{d} u_{i}=\frac{D_{i}(t)}{\Delta_{i}(\tau)} \mathrm{d} t
$$

It follows then

$$
\frac{\partial c_{i}}{\partial t}=\frac{\partial c_{i}}{\partial u_{i}} \frac{\partial u_{i}}{\partial t}=\frac{\partial c_{i}}{\partial u_{i}} \frac{D_{i}(t)}{\Delta_{i}(\tau)}=D_{i}(t) \frac{\partial^{2} c_{i}}{\partial z_{i}^{2}}
$$

When the activation energies $B_{i}$ are the same for all layers, it follows

$$
u_{i}(t)=u_{i+1}(t) \forall i
$$

and the problem to solve becomes then

$$
\begin{aligned}
& \frac{\partial c_{i}\left(z_{i}, u\right)}{\partial u}=\Delta_{i}(\tau) \frac{\partial^{2} c_{i}\left(z_{i}, u\right)}{\partial z_{i}^{2}} \\
& \forall 0<z_{i}<e_{i}, u>0 \\
& \forall i, i=1, \ldots, n \\
& c_{i}\left(z_{i}, 0\right)=0 \\
& c_{i}\left(e_{i}, u\right)=\alpha_{i, i+1} c_{i+1}(0, u) \forall i, i=1 \ldots n-1 \\
& \Delta_{i}(\tau) \frac{\partial c_{i}\left(e_{i}, u\right)}{\partial z_{i}}=\Delta_{i+1}(\tau) \frac{\partial c_{i+1}(0, u)}{\partial z_{i+1}} \forall i, i=1 \ldots n-1 \\
& c_{1}(0, u)=c_{a}(u) \\
& c_{n}\left(e_{n}, u\right)=c_{b}(u)
\end{aligned}
$$

where $c_{a}(u)$ and $c_{b}(u)$ are cyclical with period 1 . Boundary conditions depend on $u$ and have to be converted into analogous conditions independent of time. In order to do so, the so-called average mobile concentration can be introduced

$$
\hat{c}_{i}\left(z_{i}, u\right)=\int_{u-1}^{u} c_{i}\left(z_{i}, q\right) \mathrm{d} q
$$

from which

$$
\begin{aligned}
& \hat{c}_{1}(0, u)=\int_{u-1}^{u} c_{a}(q) \mathrm{d} q=\int_{0}^{1} c_{a}(q) \mathrm{d} q=\hat{c}_{a} \\
& \hat{c}_{n}\left(e_{n}, u\right)=\int_{u-1}^{u} c_{b}(q) \mathrm{d} q=\int_{0}^{1} c_{b}(q) \mathrm{d} q=\hat{c}_{b}
\end{aligned}
$$

The problem to solve becomes

$$
\begin{aligned}
& \frac{\partial \hat{c}_{i}\left(z_{i}, u\right)}{\partial u}=\Delta_{i}(\tau) \frac{\partial^{2} \hat{c}_{i}\left(z_{i}, u\right)}{\partial z_{i}^{2}} \\
& \forall 0 \leq z_{i} \leq e_{i} u>0 \\
& \forall i, i=1, \ldots, n
\end{aligned}
$$

$$
\begin{aligned}
\hat{c}_{i}\left(z_{i}, 0\right) & =0 \\
\hat{c}_{i}\left(e_{i}, u\right) & =\alpha_{i, i+1} \hat{c}_{i+1}(0, u) \forall i, i=1 \ldots n-1 \\
\Delta_{i}(\tau) \frac{\partial \hat{c}_{i}\left(e_{i}, u\right)}{\partial z_{i}} & =\Delta_{i+1}(\tau) \frac{\partial \hat{c}_{i+1}(0, u)}{\partial z_{i+1}} \forall i, i=1 \ldots n-1 \\
\hat{c}_{1}(0, u) & =\hat{c}_{a}(u)=\int_{0}^{1} c_{a}(q) \mathrm{d} q \\
\hat{c}_{n}\left(e_{n}, u\right) & =\hat{c}_{b}(u)=\int_{0}^{1} c_{b}(q) \mathrm{d} q
\end{aligned}
$$

Introducing adimensional variables

$$
\begin{aligned}
\overline{\hat{c}}_{i} & =\frac{\hat{c}_{i}}{c_{0}}, \overline{\hat{c}}_{a}=\frac{\hat{c}_{a}}{c_{0}}, \overline{\hat{c}}_{b}=\frac{\hat{c}_{b}}{c_{0}}, \bar{z}_{i}=\frac{z_{i}}{e}, \bar{e}_{i}=\frac{e_{i}}{e} \\
\bar{\Delta}_{i} & =\frac{\Delta_{i}}{e^{2}} \\
\bar{u} & =\frac{D_{0} u}{e^{2}}
\end{aligned}
$$

Then

$$
\begin{aligned}
& \frac{\partial \overline{\hat{c}}_{i}\left(\bar{z}_{i}, \bar{u}\right)}{\partial \bar{u}}=\bar{\Delta}_{i}(\tau) \frac{\partial^{2} \overline{\hat{c}}_{i}\left(\bar{z}_{i}, \bar{u}\right)}{\partial \bar{z}_{i}^{2}} \\
& \forall 0<\bar{z}_{i}<\bar{e}_{i} \bar{u}>0 \\
& \forall i, i=1, \ldots, n \\
& \overline{\hat{c}}_{i}\left(\bar{z}_{i}, 0\right)=0 \\
& \overline{\hat{c}}_{i}\left(\bar{e}_{i}, \bar{u}\right)=\alpha_{i, i+1} \overline{\hat{c}}_{i+1}(0, \bar{u}) \forall i, i=1, \ldots, n-1 \\
& \bar{\Delta}_{i}(\tau) \frac{\partial \overline{\hat{c}}_{i}\left(\bar{e}_{i}, \bar{u}\right)}{\partial \bar{z}_{i}}=\bar{\Delta}_{i+1}(\tau) \frac{\partial \overline{\hat{c}}_{i+1}(0, \bar{u})}{\partial \bar{z}_{i+1}} \forall i, i=1, \ldots, n-1 \\
& \overline{\hat{c}}_{1}(0, \bar{u})=\overline{\hat{c}}_{a}(\bar{u}) \\
& \overline{\hat{c}}_{n}\left(\bar{e}_{n}, \bar{u}\right)=\overline{\hat{c}}_{b}(\bar{u})
\end{aligned}
$$

Boundary conditions do not depend anymore on time $(t$ or $u$ ); therefore, the problem can be solved in a relatively simple way. In the Laplace domain, the solution of equations (14) and (15) takes the form

$$
\overline{\hat{c}}_{i}^{\text {Lapl }}\left(\bar{z}_{i}, s\right)=A_{i} \cos q_{i}^{2} \bar{z}_{i}+B_{i} \sin q_{i}^{2} \bar{z}_{i}
$$

where $\overline{\hat{c}}_{i}^{\text {Lapl }}\left(\bar{z}_{i}, s\right)$ is the Laplace transform of the solution

$$
\begin{gathered}
\overline{\hat{c}}_{i}^{L a p l}\left(\bar{z}_{i}, s\right)=L\left(\overline{\hat{c}}_{i}\left(\bar{z}_{i}, \bar{u}\right)\right) \int_{0}^{\infty} \overline{\hat{c}}_{i}\left(\bar{z}_{i}, \bar{u}\right) \exp (-s \bar{u}) \mathrm{d} \bar{u} \\
q_{i}^{2}=\frac{-s}{\bar{\Delta}_{i}}
\end{gathered}
$$


or

$$
q_{i}^{2}=\omega^{2} \beta_{i}^{2}
$$

where

$$
\begin{aligned}
\omega^{2} & =-s \\
\beta_{i} & =\sqrt{\frac{1}{\Delta_{i}(\tau)}}=\sqrt{\frac{e^{2}}{\Delta_{i}(\tau)}}
\end{aligned}
$$

Boundary and interface conditions in the Laplace domain now read

$$
\begin{aligned}
& \overline{\hat{c}}_{i}^{\text {Lapl }}\left(\bar{e}_{i}\right)=\alpha_{i, i+1} \overline{\hat{c}}_{i+1}^{\text {Lapl }}(0), \forall i, i=1, \ldots, n-1 \\
& \bar{\Delta}_{i} \frac{\partial \overline{\hat{c}}_{i}^{\text {Lapl }}\left(\bar{e}_{i}\right)}{\partial \bar{z}_{i}}=\bar{\Delta}_{i+1} \frac{\partial \overline{\hat{c}}_{i+1}^{\text {Lapl }}(0)}{\partial \bar{z}_{i+1}} \forall i, i=1, \ldots, n-1 \\
& \overline{\hat{c}}_{1}^{\text {Lapl }}(0)-\frac{\overline{\hat{c}}_{a}}{S}=0 \\
& \overline{\hat{c}}_{n}^{\text {Lapl }}\left(\bar{e}_{n}\right)-\frac{\overline{\hat{c}}_{b}}{S}
\end{aligned}
$$

By applying the theorem of residues, the solution of the problem in the time domain is

$$
\begin{aligned}
\overline{\hat{c}}_{i}\left(\bar{z}_{i}, \bar{u}\right)= & \frac{1}{\Delta_{s}}\left(A_{i}^{\prime}+B_{i}^{\prime} \bar{z}_{i}\right)+\sum_{m=1}^{\infty} \frac{2 \exp \left(-\omega_{m}^{2} \bar{u}\right)}{\omega_{m} \Delta_{u}^{\prime}\left(\omega_{m}\right)} \\
& \left\{A_{i}^{*}\left(\omega_{m}\right) \cos \left(\beta_{i} \omega_{m} \bar{z}_{i}\right)+B_{i}^{*}\left(\omega_{m}\right) \sin \left(\beta_{i} \omega_{m} \bar{z}_{i}\right)\right\}
\end{aligned}
$$

where $\Delta_{u}$ and $\Delta_{s}$ are the determinants of the $2 n \times 2 n(a)$ and (b) matrices (equations (23) and (24)).

$A_{i}^{*}\left(\omega_{m}\right)$ and $B_{i}^{*}\left(\omega_{m}\right)$ are the determinants calculated in $\omega_{m}\left(\omega_{m}\right.$ in the $m$ th positive root of the equation $\left.\Delta_{u}(\omega)=0\right)$ of the matrices with components $a_{i j}$ in which the columns $(2 i-1)$ and $2 i$ are substituted by the constant vector $\{g\}$ (equation (25)). $A_{i}^{\prime}$ and $B_{i}^{\prime}$ are the determinants calculated in $\omega_{m}$ of the matrices with components $a_{i j}$ in which the columns $(2 i-1)$ and $2 i$ are substituted by the constant vector $\{g\}$. Finally, $\Delta_{u}^{\prime}\left(\omega_{m}\right)$ is the derivative of $\Delta_{u}$ with respect to $\omega$ and evaluated at $\omega_{m}$.

Non-zero elements of matrices $(a)$ and $(b)$ and of vector $\{g\}$ are given by, for $i=1 \ldots n-1$

$$
\begin{aligned}
a_{11}= & 1 \\
a_{2 n 2 n-1}= & \cos \beta_{n} \omega \bar{e}_{n}, a_{2 n 2 n}=\sin \beta_{n} \omega \bar{e}_{n} \\
a_{2 i 2 i-1}= & \cos \beta_{i} \omega \bar{e}_{i}, a_{2 i 2 i}=\sin \beta_{i} \omega \bar{e}_{i}, a_{2 i 2 i+1}=-\alpha_{i, i+1} \\
a_{2 i+12 i-1}= & -\bar{\Delta}_{i} \beta_{i} \sin \beta_{i} \omega \bar{e}_{i}, a_{2 i+12 i}=\bar{\Delta}_{i} \beta_{i} \cos \beta_{i} \omega \bar{e}_{i}, \\
& a_{2 i+12 i+2}=-\bar{\Delta}_{i+1} \beta_{i+1}
\end{aligned}
$$

$$
\begin{aligned}
b_{11}= & 1 \\
b_{2 n 2 n-1}= & 1, b_{2 n 2 n}=\bar{e}_{n} \\
b_{2 i 2 i-1}= & 1, b_{2 i 2 i}=\bar{e}_{i}, b_{2 i 2 i+1}=-\alpha_{i, i+1} \\
b_{2 i+12 i}= & \bar{\Delta}_{i}, b_{2 i+12 i+2}=-\bar{\Delta}_{i+1} \\
& g_{1}=\overline{\hat{c}}_{a}, g_{2 n}=\overline{\hat{c}}_{b}
\end{aligned}
$$

For a homogeneous plate (with respect to water diffusion), matrices $(a)$ and $(b)$ become

$$
\begin{gathered}
{[a]=\left[\begin{array}{cc}
1 & 0 \\
\cos \beta_{n} \omega \bar{e}_{n} & \sin \beta_{n} \omega \bar{e}_{n}
\end{array}\right]} \\
{[b]=\left[\begin{array}{cc}
1 & 0 \\
1 & \bar{e}_{n}
\end{array}\right]}
\end{gathered}
$$

It is worth noting that the solution of the hygroscopic problem in its analytic form (equation (22)) is formally analogous to that of a laminated cylinder under the same conditions (see Carslaw and Jaeger; ${ }^{6}$ see also Ootao et al. ${ }^{14}$ and Tanigawa et al. ${ }^{15}$ for pure thermal transient problems). In that case, however, the linear term of the stationary solution is replaced by an equivalent logarithmic term, while functions, $\sin / \cos$ of the transient solution are replaced by Bessel functions of zeroth order. In the sequel, equation (22) will be expressed as a function of the number of cycles by adopting a recursive technique.

Since

$$
\overline{\hat{c}}_{i}\left(\bar{z}_{i}, \bar{u}\right)=\int_{\bar{u}-1}^{\bar{u}} \bar{c}_{i}\left(\bar{z}_{i}, q\right) \mathrm{d} q
$$

it follows

$$
\bar{c}_{i}\left(\bar{z}_{i}, \bar{u}\right)=\frac{\partial \overline{\hat{c}}_{i}\left(\bar{z}_{i}, \bar{u}\right)}{\partial \bar{u}}+\bar{c}_{i}\left(\bar{z}_{i}, \bar{u}-1\right)
$$

If $N$ is the number of cycles at saturation, then

$$
\bar{c}_{i}\left(\bar{z}_{i}, N+1\right)=\bar{c}_{i}\left(\bar{z}_{i}, N\right)
$$

$$
\frac{\partial \overline{\hat{c}}_{i}\left(\bar{z}_{i}, N+1\right)}{\partial \bar{u}}=0 \Rightarrow \overline{\hat{c}}_{i}\left(\bar{z}_{i}, N+1\right)=\frac{1}{\Delta_{s}}\left(A_{i}^{\prime}+B_{i}^{\prime} \bar{z}_{i}\right)
$$

$\overline{\hat{c}}_{i}\left(\bar{z}_{i}, N+1\right)$ is defined by

$$
\begin{gathered}
\overline{\hat{c}}_{i}\left(\bar{z}_{i}, N+1\right)=\int_{N}^{N+1} \bar{c}_{i}\left(\bar{z}_{i}, u\right) \mathrm{d} u=\bar{c}_{i}\left(\bar{z}_{i}, N\right) \\
\bar{c}_{i}\left(\bar{z}_{i}, N\right)=\frac{1}{\Delta_{s}}\left(A_{i}^{\prime}+B_{i}^{\prime} \bar{z}_{i}\right)
\end{gathered}
$$


but it is also

$$
\begin{aligned}
\bar{c}_{i}\left(\bar{z}_{i}, N\right) & =\frac{\partial \overline{\hat{c}}_{i}(z, N)}{\partial \bar{u}}+\bar{c}_{i}\left(\bar{z}_{i}, N-1\right) \\
\bar{c}_{i}\left(\bar{z}_{i}, N-k\right) & =\frac{\partial \overline{\hat{c}}_{i}\left(\bar{z}_{i}, N-k\right)}{\partial \bar{u}}+\bar{c}_{i}\left(\bar{z}_{i}, N-k-1\right) \\
\bar{c}_{i}\left(\bar{z}_{i}, N-k\right) & =\bar{c}_{i}\left(\bar{z}_{i}, N\right)-\frac{\partial \overline{\hat{c}}_{i}\left(\bar{z}_{i}, N\right)}{\partial \bar{u}}-\cdots-\frac{\partial \overline{\hat{c}}_{i}\left(\bar{z}_{i}, N-k+1\right)}{\partial \bar{u}}
\end{aligned}
$$

and

$$
\begin{aligned}
\frac{\partial \overline{\hat{c}}_{i}\left(\bar{z}_{i}, N\right)}{\partial \bar{u}}= & -\sum_{m=1}^{\infty} \omega_{m}^{2} \frac{2 \exp \left(-\omega_{m}^{2} N\right)}{\omega_{m} \Delta_{u}^{\prime}\left(\omega_{m}\right)}\left\{A_{i}^{*}\left(\omega_{m}\right) \cos \left(\beta_{i} \omega_{m} \bar{z}_{i}\right)\right. \\
& \left.+B_{i}^{*}\left(\omega_{m}\right) \sin \left(\beta_{i} \omega_{m} \bar{z}_{i}\right)\right\}
\end{aligned}
$$$$
\frac{\partial \overline{\hat{c}}_{i}\left(\bar{z}_{i}, N-k+1\right)}{\partial \bar{u}}=-\sum_{m=1}^{\infty} \omega_{m}^{2} \frac{2 \exp \left(-\omega_{m}^{2}(N-k+1)\right)}{\omega_{m} \Delta_{u}^{\prime}\left(\omega_{m}\right)}
$$$$
\times\left\{A_{i}^{*}\left(\omega_{m}\right) \cos \left(\beta_{i} \omega_{m} \bar{z}_{i}\right)+B_{i}^{*}\left(\omega_{m}\right) \sin \left(\beta_{i} \omega_{m} \bar{z}_{i}\right)\right\}
$$

Finally

$$
\begin{aligned}
\overline{\hat{c}}_{i}\left(\bar{z}_{i}, N-k\right)= & \frac{1}{\Delta_{s}}\left(A_{i}^{\prime}+B_{i}^{\prime} \bar{z}_{i}\right)+\sum_{m=1}^{\infty} \omega_{m}^{2} \frac{2 \exp \left(-\omega_{m}^{2} N\right)}{\omega_{m} \Delta_{u}^{\prime}\left(\omega_{m}\right)} \\
& \times\left\{A_{i}^{*}\left(\omega_{m}\right) \cos \left(\beta_{i} \omega_{m} \bar{z}_{i}\right)+B_{i}^{*}\left(\omega_{m}\right) \sin \left(\beta_{i} \omega_{m} \bar{z}_{i}\right)\right\} \\
& +\cdots+ \\
& \sum_{m=1}^{\infty} \omega_{m}^{2} \frac{2 \exp \left(-\omega_{m}^{2}(N-k+1)\right)}{\omega_{m} \Delta_{u}^{\prime}\left(\omega_{m}\right)} \\
& \times\left\{A_{i}^{*}\left(\omega_{m}\right) \cos \left(\beta_{i} \omega_{m} \bar{z}_{i}\right)+B_{i}^{*}\left(\omega_{m}\right) \sin \left(\beta_{i} \omega_{m} \bar{z}_{i}\right)\right\}
\end{aligned}
$$

After $(N-k)$ cycles

$$
\begin{aligned}
\overline{\hat{c}}_{i}\left(\bar{z}_{i}, N-k\right)= & \frac{1}{\Delta_{s}}\left(A_{i}^{\prime}+B_{i}^{\prime} \bar{z}_{i}\right) \\
& +\sum_{m=1}^{\infty} \frac{2 \omega_{m}}{\Delta_{u}^{\prime}\left(\omega_{m}\right)}\left[\sum_{j=0}^{k-1} \exp \left(-\omega_{m}^{2}(N-j)\right)\right] \\
& \times\left\{A_{i}^{*}\left(\omega_{m}\right) \cos \left(\beta_{i} \omega_{m} \bar{z}_{i}\right)+B_{i}^{*}\left(\omega_{m}\right) \sin \left(\beta_{i} \omega_{m} \bar{z}_{i}\right)\right\}
\end{aligned}
$$

In equation (38), boundary conditions and diffusion coefficients are constant; therefore, the solution of equation (38) is valid only at a certain distance from the surface boundaries. In other words, since the analytical solution of equation (38) employs average boundary conditions and diffusion coefficients, fluctuations close to the external surfaces cannot be simulated by this solution. The fluctuating part of the solution can be simulated by finite difference methods. ${ }^{5}$

\section{Homogeneous plate}

In the case of a homogeneous plate, it can be shown that the distance at which the analytical solution becomes applicable is given by (see also Verchery $^{16}$ )

$$
e_{0}=2 \sqrt{\pi \int_{0}^{\tau} D(t) \mathrm{d} t}
$$

which is then the characteristic length within which external fluctuations are effective. It is interesting to note that this characteristic length is analogous to that given by Kelvin ${ }^{17}$ for the case of temperature fluctuations on the hearth surface temperature induced by solar radiation. Here, $e_{0}$ depends on the material, through the diffusion coefficient $D(t)$, and on the period of a cycle $\tau$.

For a homogeneous plate with diffusion coefficient $D(t)$, the change in variable is then

$$
\begin{aligned}
\Delta(\tau) & =\int_{0}^{\tau} D(t) \mathrm{d} t \\
\hat{c}_{a} & =\frac{1}{\Delta(\tau)} \int_{0}^{\tau} D(t) c_{a}(t) \mathrm{d} t \\
\hat{c}_{b} & =\frac{1}{\Delta(\tau)} \int_{0}^{\tau} D(t) c_{b}(t) \mathrm{d} t
\end{aligned}
$$

For a homogeneous plate with identical boundary conditions $\hat{c}_{\infty}$ on the two lateral surfaces, after $(N-k)$ cycles

$$
\begin{aligned}
c(z, N-k)= & \hat{c}_{\infty}-\frac{4 \hat{c}_{\infty} \pi}{\beta^{2}} \sum_{n=0}^{\infty} \sum_{i=0}^{k-1}\left((-1)^{n}(2 n+1)\right. \\
& \left.\times \exp \left(-\frac{(2 n+1)^{2} \pi^{2}(N-i)}{\beta^{2}}\right)\right) \cos \left(\frac{(2 n+1) \pi z}{e}\right) \\
& \forall\left(-\frac{e}{2}\right)<z<\left(\frac{e}{2}\right)
\end{aligned}
$$

where

$$
\beta=\sqrt{\frac{e^{2}}{\Delta(\tau)}}
$$


When the initial concentration $c_{i}(z, 0)$ is different than zero, then

$$
\begin{aligned}
c(z, N-k)= & \hat{c}_{\infty}+\frac{\Delta(\tau) \pi^{2}}{2\left(\frac{e}{2}\right)^{3}} \sum_{n=0}^{\infty} \sum_{i=0}^{k-1}\left((2 n+1)^{2}\right. \\
& \left.\left.\times \exp -\frac{(2 n+1)^{2} \pi^{2} \Delta(\tau)(N-i)}{4\left(\frac{e}{2}\right)^{2}}\right)\right) \\
& \times \cos \left(\frac{(2 n+1) \pi z}{e}\right)\left(\frac{2\left(\frac{e}{2}\right)(-1)^{n+1} \hat{c}_{\infty}}{(2 n+1) \pi}\right. \\
& \left.\left.+\int_{0}^{e / 2} c_{i}\left(z^{\prime}\right) \frac{(2 n+1) \pi z^{\prime}}{2\left(\frac{e}{2}\right)}\right) \mathrm{d} z^{\prime}\right) \\
& \forall\left(-\frac{e}{2}\right)<z<\left(\frac{e}{2}\right)
\end{aligned}
$$

It is interesting to note that the analytical solutions given by equations (22), (41) and (43) can be subjected to truncation errors of the series, in particular for a low number of cycles, that is, far from saturation.

In order to schematically and qualitatively illustrate the problem and its solutions, let us consider a homogeneous plate with thickness $e$ subjected to cyclical hygrothermal conditions on the two external lateral surfaces, see Figure 1.

Assuming unidirectional diffusion from a physical point of view means that the plate has a length and a width much bigger than its thickness; in other words, the length and the width of the plate are assumed to be infinite. Environmental conditions (temperature and $\mathrm{RH})$ vary, for instance, as illustrated in Figure 2, temperature varies between $T_{0}$ and $T_{1}$ (Figure 2(a)) and the $\mathrm{RH}$ of the external environment (in \%) between $R H_{0}$ and $R H_{1}$ (Figure 2(b)). Fluctuations are periodic of period $\tau$. Fluctuations of RH induce water concentration fluctuations on the external surfaces of the plate following, for instance, the law

$$
c(t)=C(R H(t))^{b}
$$

where $C$ and $b$ are material constants, while the diffusion coefficient varies as a function of temperature as stated, for instance, by equation (3).

As mentioned, it is assumed that at each time the temperature is uniform within the homogeneous plate: this approximation is admissible when considering that thermal and water diffusion transfers differ by many orders of magnitude.

The distribution of water concentration $c(t)$ within the homogeneous plate is schematically illustrated in Figure 3, in which the schematic results of finite difference simulations are also reported.

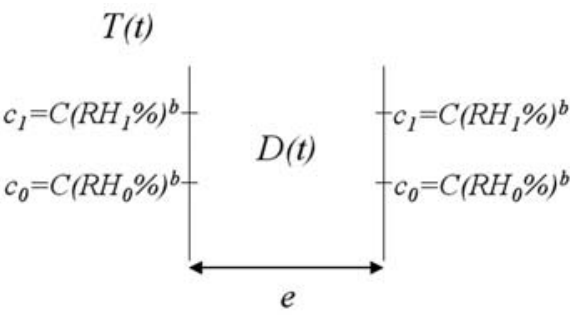

Figure I. Schematic representation of a homogeneous plate subjected to environmental temperature and relative humidity cyclical fluctuations at its external lateral boundaries.

Water concentration fluctuates at the external lateral boundaries between $c_{0}$ and $c_{1}$, the extent of the fluctuating zone within the plate is equal to $e_{0}$. The fluctuating part of the solution can be simulated by finite difference methods. The analytical solution, on the other hand, employs constant average boundary conditions, namely

$$
\hat{c}_{\infty}=\frac{1}{\Delta(\tau)} \int_{0}^{\tau} D(t) c_{\infty}(t) \mathrm{d} t
$$

In the transient state (after $n$ cycles), zones close to the external surfaces are influenced by external fluctuations with period $\tau$, the extent of the fluctuating zone is $e_{0}$, while internal zones are in a transient regime: in the steady state ( $N$ cycles), water concentration is uniform along almost all the thickness of the plate with value $\hat{c}_{\infty}$; fluctuations persist along $e_{0}$, that is, in zones close to the external surfaces, and are still of period $\tau$. According to equation (39), the extent of the fluctuating zone, $e_{0}$, is constant with increasing number of cycles.

\section{Application to aircraft plate structures subjected to supersonic flight environments}

An important practical application of the proposed method concerns aircraft structures. These are typically subjected to environmental conditions of almost constant period, which is the time of a flight.

Figure 4 presents schematically a hygrothermal cycle which is related to supersonic flight conditions and to which supersonic aircraft structures are subjected. Point A represents the start of a cycle/flight (ground conditions: $23^{\circ} \mathrm{C}, \mathrm{RH} 50 \%$ ), B the start of a subsonic phase, characterised by low temperatures and RH (typically $-55^{\circ} \mathrm{C}, \mathrm{RH} 0 \%$ ). Point $\mathrm{C}$ represents the start of a supersonic phase: the exposed structures are subjected to high temperatures due to aerodynamic friction (typically $130^{\circ} \mathrm{C}$ ) and to low RH. Finally, point D represents the end of a cycle/flight.

Plate hygroscopic properties used for simulations are reported in Table 1: they are representative of an 
(a)

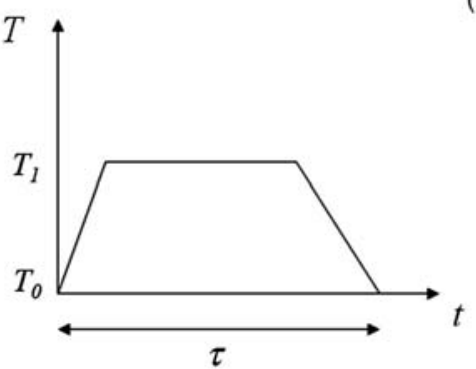

(b)

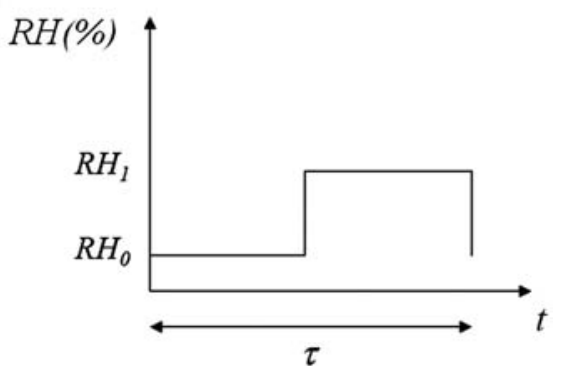

Figure 2. Schematic example of cyclical temperature (a) and relative humidity (b) fluctuations at the external lateral boundaries of the plate.
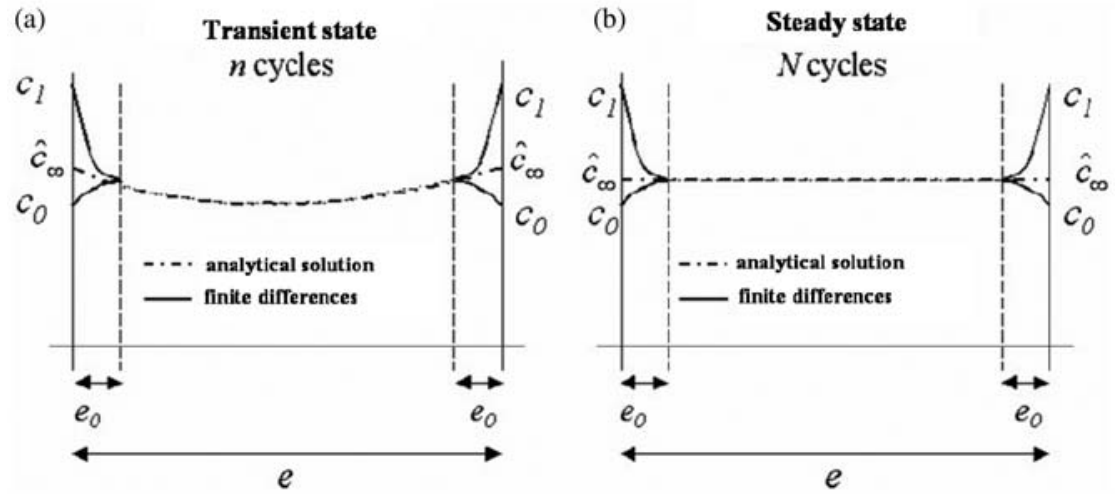

Figure 3. Schematic representation of the water concentration solution $c(t)$ : (a) transient regime ( $n$ cycles) and (b) permanent regime ( $N$ cycles).

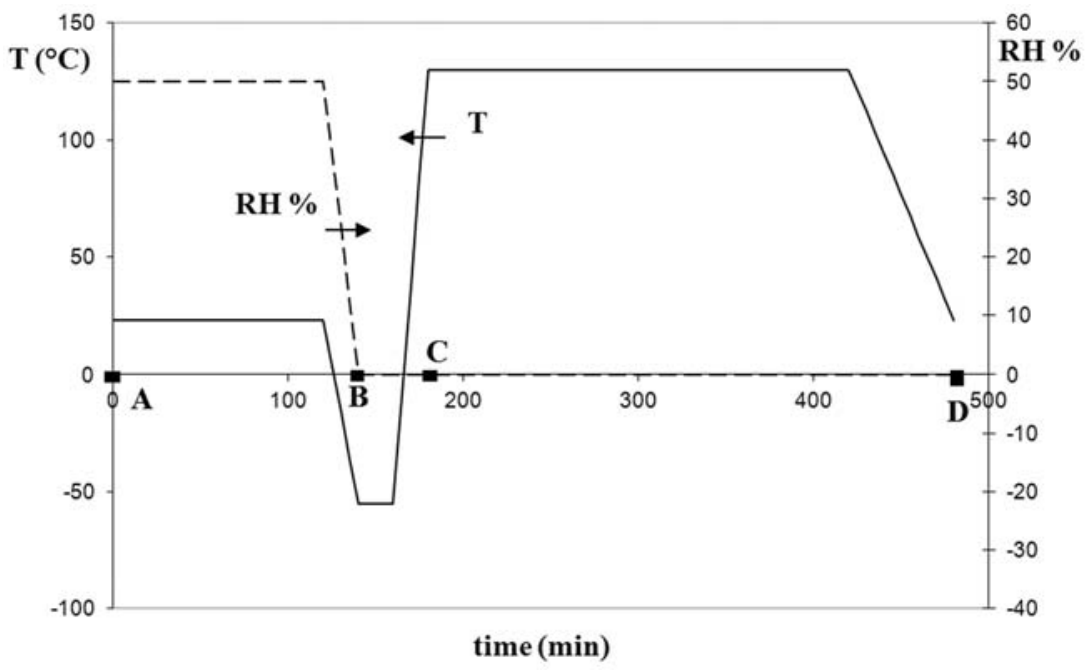

Figure 4. Hygrothermal cycle for a structure exposed to a supersonic flight environment.

IM7/977-2 carbon-epoxy composite material, which is considered as an equivalent homogeneous material from the hygroscopic point of view. ${ }^{18}$ It is supposed that the diffusive properties of the composite laminate are constant along its thickness; in other words, the laminate is homogeneous with respect to water diffusion along the thickness direction. This hypothesis which seems restrictive at first sight - is plausible not 
Table I. Plate hygroscopic properties

\begin{tabular}{llll}
\hline$A$ (equation $(3))\left(\mathrm{mm}^{2} / \mathrm{h}\right)$ & $B($ equation $(3))\left(\mathrm{K}^{-1}\right)$ & $C$ (equation $(44))(\%)$ & $B$ (equation $(44))$ \\
\hline 7.18 & -2910 & 0.0007 & 1.60 \\
\hline
\end{tabular}

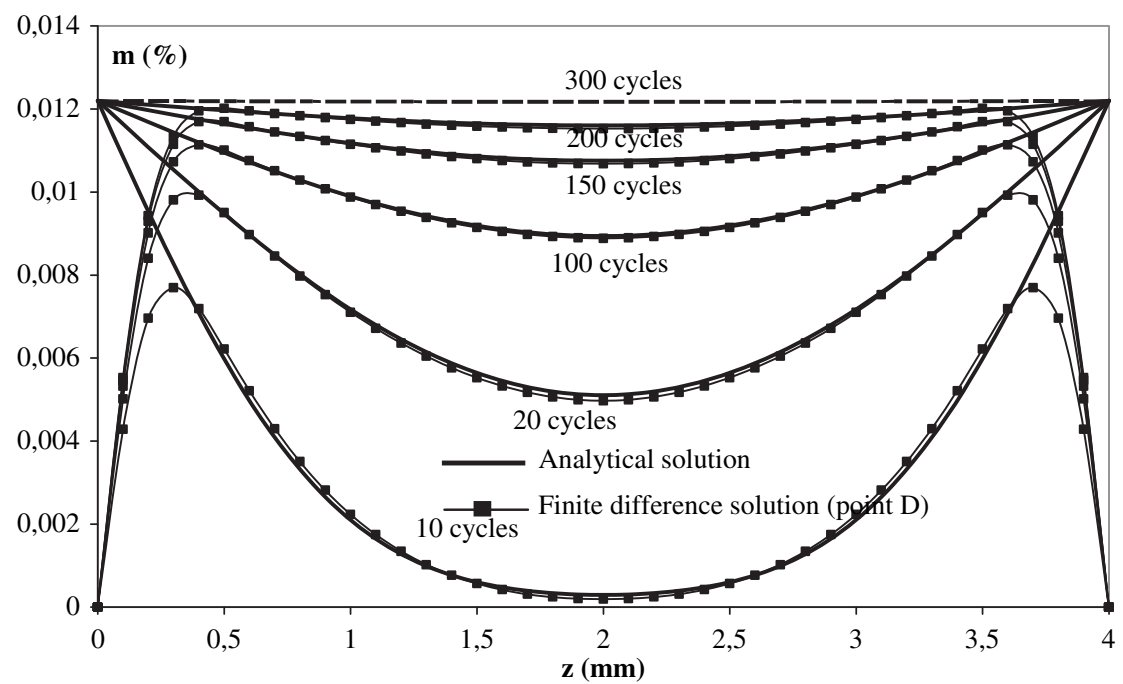

Figure 5. Water composition profile along the thickness of a $4 \mathrm{~mm}$ composite plate during supersonic cycling at different numbers of cycles (initial state: dry, $m(z)=0 \%$ ).

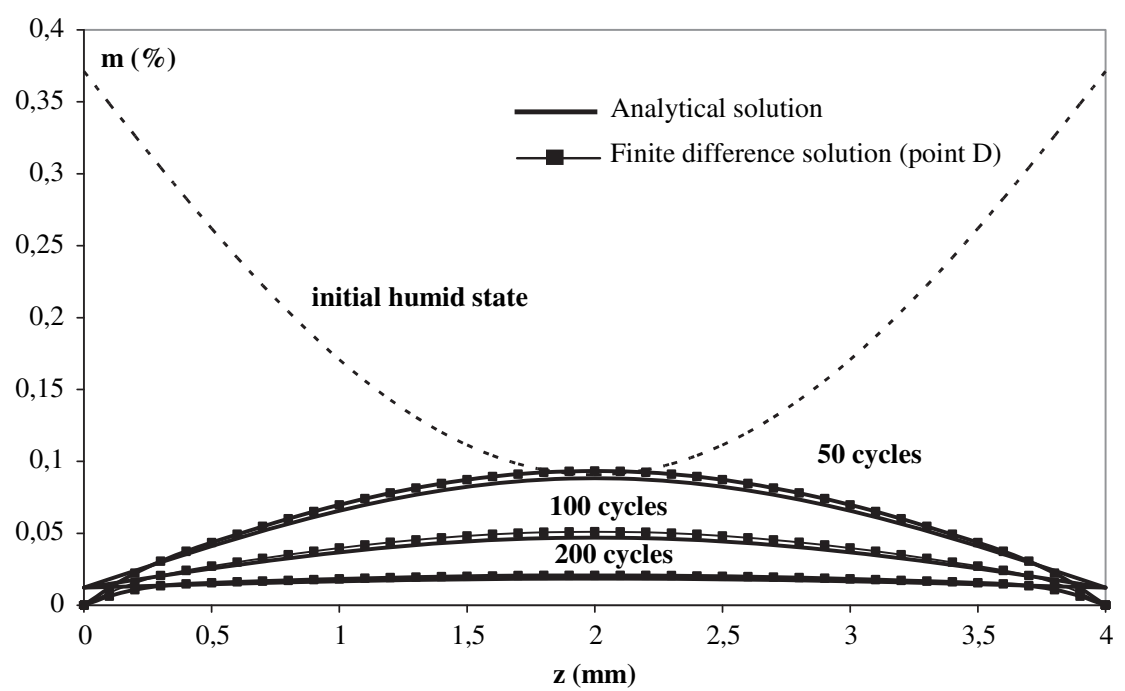

Figure 6. Water composition profile along the thickness of a $4 \mathrm{~mm}$ composite plate during supersonic cycling at different numbers of cycles (initial state: wet, $m(z)$ : heterogeneous initial humid state).

only for unidirectional laminates but also for laminates with complex stacking sequences, ${ }^{1,2}$ provided the laminae are made of the same composite material.

Figure 5 presents water composition profiles $\left(m(z)=c(z) / \rho_{s}\right.$, where $\rho_{s}$ is the density of the dry material, in the present case $\rho_{s}=1500 \mathrm{~kg} / \mathrm{m}^{3}$ ) along the thickness of a $4 \mathrm{~mm}$ composite plate after 10, 20, 100, 150,
200 and 300 supersonic cycles (end of the cycle, point D) for both finite difference and analytical solutions. In this case, the plate is initially in a dry state $(m(z)=0 \%$ after 0 cycles) and tends towards a uniform saturated state $(m(z) \approx 0.012 \%)$ after 300 cycles. The analytical solution employs average boundary conditions - given by equation (45) - which also represent the uniformly 


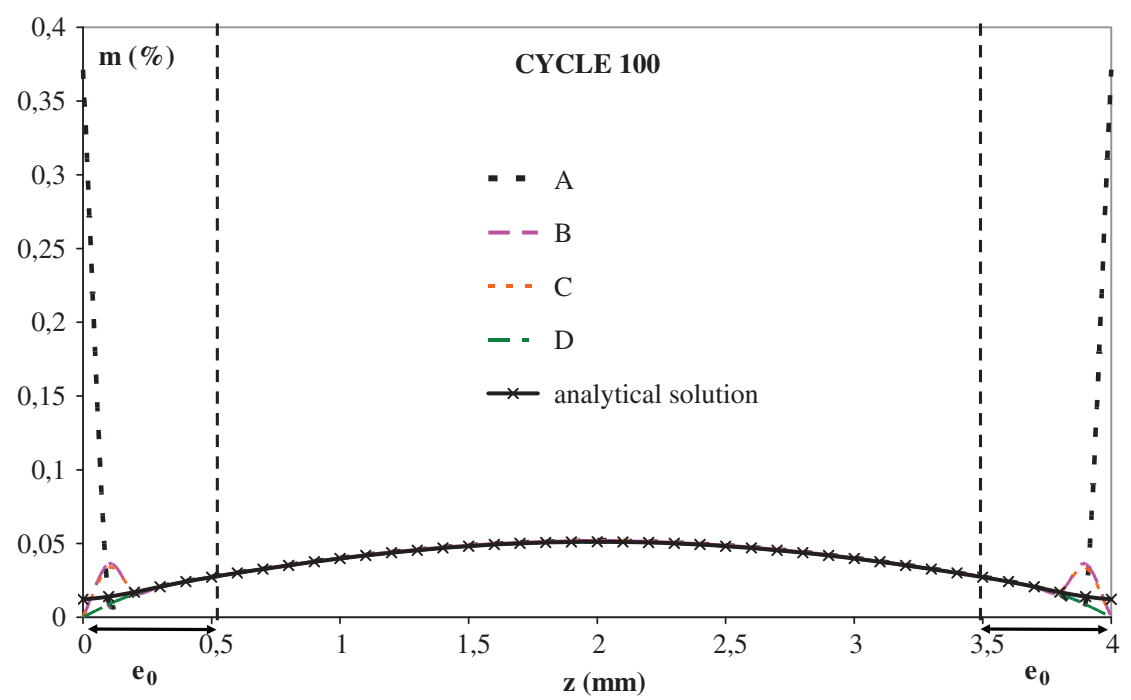

Figure 7. Water composition profile along the thickness of a $4 \mathrm{~mm}$ composite plate during supersonic cycling at cycle 100 (initial state: wet, $m(\mathbf{z})$ : heterogeneous initial humid state).

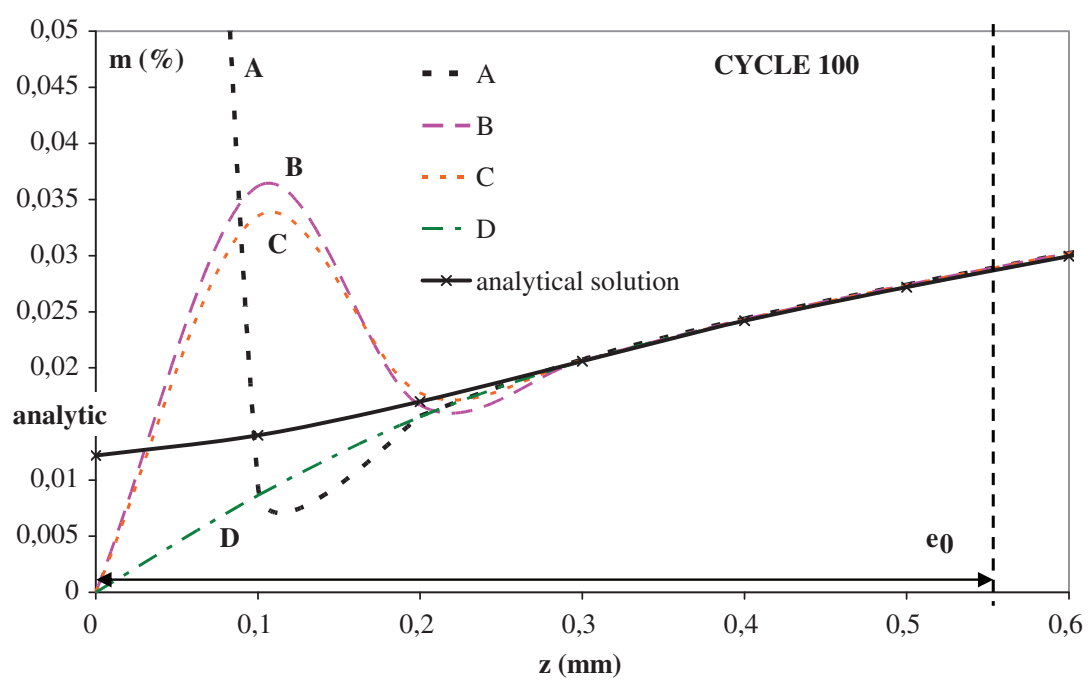

Figure 8. Detail of the water composition profile along the thickness of a $4 \mathrm{~mm}$ composite plate during supersonic cycling at cycle 100 (initial state: wet, $m(z)$ : heterogeneous initial humid state).

distributed composition at saturation. The finite difference solution captures the fluctuations occurring along a cycle and is able to reproduce exactly the composition boundary condition at any stage of the supersonic cycle. It can be seen that the analytical solution converges rapidly starting from $n=10$ cycles and is capable to well represent the transient average water composition profile along a cycle, as a function of the number of cycles. Though is not able to represent fluctuations close to the plate boundaries, the analytical solution is able to provide - through equation (39) - the extent of the fluctuating zone $e_{0}$, which is equal to $0.56 \mathrm{~mm}$ in this specific application. From Figure 5, it can be noted that the calculation of $e_{0}$ by the explicit expression, equation (39), is in agreement with finite difference simulations and is constant with increasing number of cycles.

Figure 6 presents water composition profiles along the thickness of a $4 \mathrm{~mm}$ composite plate after 50,100, 200 supersonic cycles (end of the cycle, point D) for both finite difference and analytical solutions. In this case - at the start of the supersonic cycling - the plate is in a wet state (initial humid state, dotted line in Figure 6): this non-uniform composition state is induced by a conditioning phase on ground $\left(23^{\circ} \mathrm{C}, \mathrm{RH} 50 \%\right.$ for 3 months) simulating the time of a long maintenance at an airport. Again, after 300 cycles, the plate tends towards a saturated state $(m(z)=0.012 \%)$, which is 


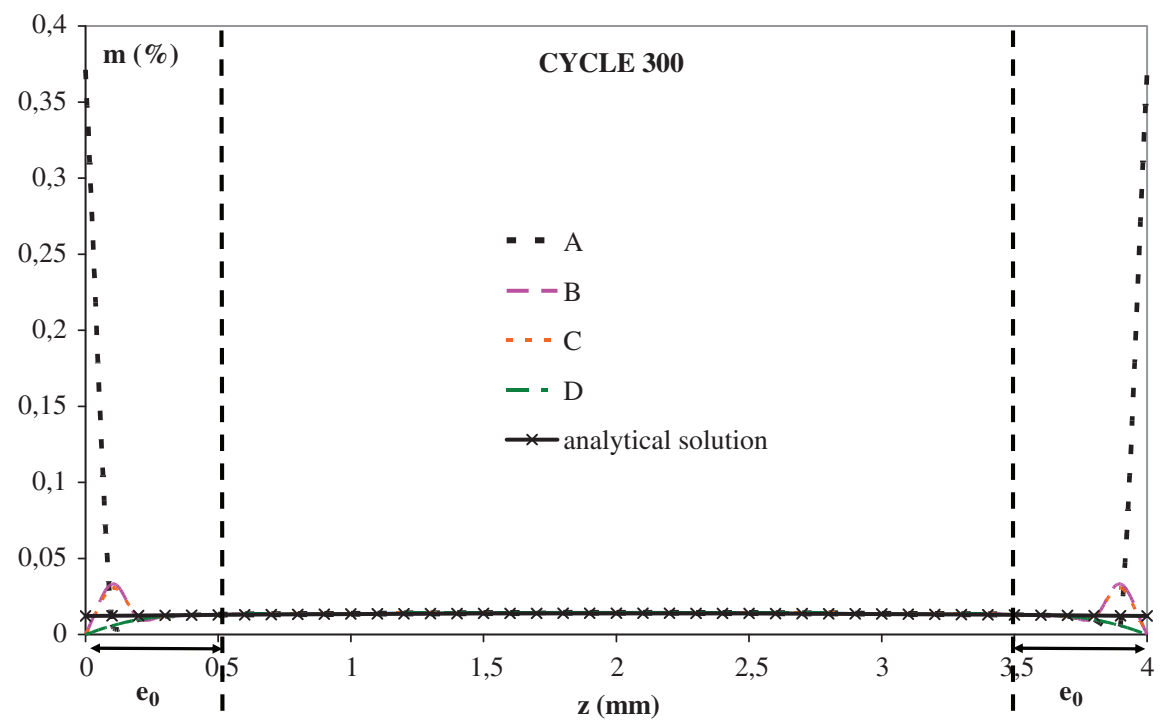

Figure 9. Water composition profile along the thickness of a $4 \mathrm{~mm}$ composite plate during supersonic cycling at cycle 300 (initial state: wet, $m(z)$ : heterogeneous initial humid state).

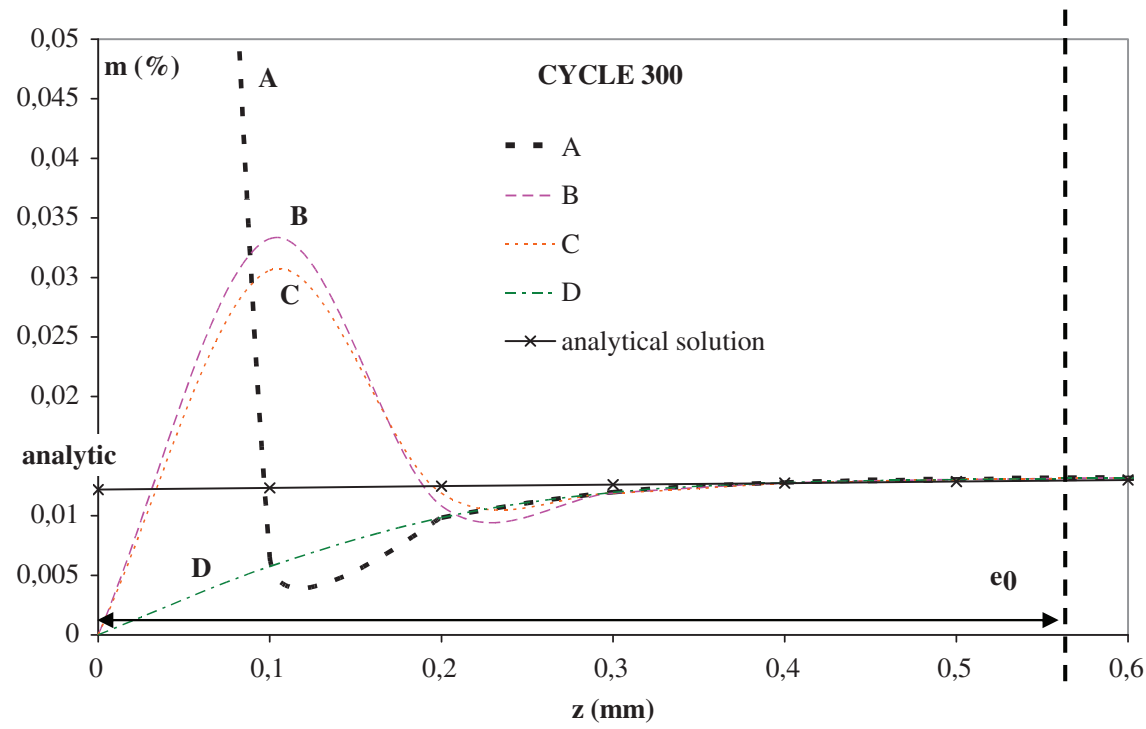

Figure 10. Detail of the water composition profile along the thickness of a $4 \mathrm{~mm}$ composite plate during supersonic cycling at cycle 300 (initial state: wet, $m(z)$ : heterogeneous initial humid state).

the same as that illustrated in Figure 5, after the same number of cycles. It is again stressed that the saturation state is provided by the analytical formula, equation (45). Thus - irrespective of its initial composition state - the plate tends always towards the same saturation state, which is given by the simple analytical expression equation (45).

Useless to say, the extent of the fluctuating zone is again equal to $0.56 \mathrm{~mm}-$ as in the case in Figure 5 and is again given by equation (39).

Figure 7 presents the water composition profiles along a $4 \mathrm{~mm}$ thick plate at different stages (points
A-D) of a supersonic flight/cycle, after 100 flight/ cycles. Both finite difference and analytical (average along a cycle) solutions are reported. According to the external environmental conditions, the water composition $m(z)$ at the plate boundaries $(z=0$ and $z=4 \mathrm{~mm}$ ) fluctuates between $m(z)=0 \%$ ('dry' section of the cycle) and around $m(z)=0.4 \%$ ('wet' section of the cycle). Fluctuations extend within a consistent zone of the structure (1/4 of the total thickness) and engender consistent concentration gradients; however, their magnitude is bounded between two a priori known values, $m(z)=0 \%$ (start of a cycle) and $m(z) \approx 0.4 \%$ 
(end of a cycle) - which can be easily determined by the systematic use of equation (44). Therefore calculating the average composition profile by the analytical solution (equation (43)), the external fluctuating boundary conditions (equation (44)) and the extent of the fluctuating zone (equation (39)) gives a first fast approximation of the composition gradient within the fluctuating zone at any number of cycles without performing finite difference calculations.

Figure 8 shows composition values during cycle 100 in a zone affected by fluctuations close to the plate boundaries, within $e_{0}$. It can be seen in greater detail that the analytical solution resumes the average composition field along a cycles. Fluctuations are confined to a zone whose extent is related to the type of hygrothermal cycle.

Similar to Figures 7 and 8, Figures 9 and 10 present the water composition profiles along a $4 \mathrm{~mm}$ thick plate at different stages (points $\mathrm{A}-\mathrm{D}$ ) of a supersonic flight/ cycle, after 300 flight/cycles and within a zone $e_{0}$ affected by fluctuations close to the plate boundaries, respectively. Again, according to the external environmental conditions, the water composition $m(z)$ at the plate boundaries $(z=0$ and $z=4 \mathrm{~mm})$ fluctuates between $m(z)=0 \%$ and around $m(z)=0.4 \%$. The behaviour is qualitatively similar to that illustrated in Figures 7 and 8 , this time the average composition reaching saturation. With respect to Figures 7 and 8 , the extent of the fluctuating zone and of the fluctuation values is almost unchanged; in some way, this can justify the interest of having at disposition analytical solution to rapidly calculate the transient average composition during the hygrothermal cycling.

\section{Conclusion and perspectives}

An analytical solution for laminated plates subjected to cyclical hygrothermal conditions has been provided for multilayer composite plates. Thermal transfers are discarded; it is assumed that temperature diffusion phenomena are much faster than water concentration diffusion. The solution can be employed as benchmark for simulations based on numerical procedures and can be employed for evaluating the induced hygrothermal stress, therefore, for structural, design and optimisation purposes. The solution is unidirectional, and therefore is applicable for slender plates. A relatively simple solution can be found for a homogeneous plate and this solution is applied to a practical case: the hygrothermal cycling related to a supersonic flight environment.

The analytical solution rapidly converges starting from $n=10$ cycles and employs average boundary conditions - given by equation (45) - which also represent the uniformly distributed composition at saturation: it is capable to well represent the transient average water composition profile along a cycle, as a function of the number of cycles. The finite difference solution captures the fluctuations occurring along a cycle and is able to exactly reproduce the composition boundary condition at any stage of the supersonic cycle. Though is not able to represent fluctuations close to the plate boundaries, the analytical solution is able to provide - through equation (39) - the extent of the fluctuating zone $e_{0}$.

Therefore calculating the average composition profile by the analytical solution (equation (43)) the external fluctuating boundary conditions (equation (44)) and the extent of the fluctuating zone (equation (39)) gives a first fast approximation of the composition gradient within the fluctuating zone at any number of cycles without performing finite difference calculations.

\section{Acknowledgements}

Part of the present research has been accomplished within the context of the Supersonique 2001 French Supersonic research program, supported by the French Ministry of Research. All partners of the research, ONERA, CCR EADS and Ecole Centrale Lyon are gratefully acknowledged.

\section{Funding}

This research received no specific grant from any funding agency in the public, commercial, or not-for-profit sectors.

\section{Conflict of interest}

None declared.

\section{References}

1. Springer GS, Sanders BA and Tung RW. Environmental effect on glass-fiber reinforced polyester and vinyl ester composites. In: Springer GS (ed.) Environmental effects of composite materials. Westport, CT: Technomic Publishing Company, 1981, pp.126-144.

2. Weitsman YJ. Effects of fluids on polymeric composites. A review. In: Talreja R and Manson JAE (eds) Polymer matrix composites. Elsevier, 2000, pp.369-401.

3. Gates TS. Durability assessment of polymeric composites for high speed civil transport. In: Proceedings of the DURACOSYS 99, Bruxelles, Belgique, 11-14 July 1999.

4. Crank J. The mathematics of diffusion, 2nd edn. London: Oxford University Press, 1975.

5. Jacquemin F and Vautrin A. Modelling of the moisture concentration field due to cyclical hygrothermal conditions in thick laminated pipes. Eur $J$ Mech A Solids 2002; 21: 845-855.

6. Carslaw HS and Jaeger JC. Conduction of heat in solids, 2nd edn. New York: Oxford University Press, 1974.

7. Benkeddad A, Grédiac M and Vautrin A. Computation of transient hygroscopic stresses in laminated composite plates. Compos Sci Technol 1996; 56: 869-876.

8. Jacquemin F and Vautrin A. A closed-form solution for the internal stresses in thick composite cylinders induced by cyclical environmental conditions. Compos Struct 2002; 58: $1-9$. 
9. Gigliotti $\mathrm{M}$, Jacquemin $\mathrm{F}$ and Vautrin A. Internal stresses in composite laminates due to cyclical hygrothermal loading. AIAA J 2004; 42: 2600-2605.

10. Gigliotti M, Jacquemin F and Vautrin A. Assessment of approximate models to evaluate transient and cyclical hygrothermoelastic stress in composite plates. Int $J$ Solids Struct 2007; 44: 733-759.

11. Weitsman YJ. Coupled damage and moisture-transport processes in fiber reinforced, polymeric composites. Int $J$ Solids Struct 1987; 23: 1003-1025.

12. Lundgren JE and Gudmundson P. A model for moisture absorption in cross-ply laminates with matrix cracks. J Compos Mater 1998; 32: 2226-2253.

13. Weitsman YJ and Guo YJ. A correlation between fluid-induced damage and anomalous fluid sorption in polymeric composites. Compos Sci Technol 2002; 62: 889-908.
14. Ootao Y, Tanigawa Y and Fukuda T. Axisymmetric transient thermal stress analysis of a multilayered composite hollow cylinder. $J$ Therm Stresses 1991; 14: 201-213.

15. Tanigawa $\mathrm{Y}$, Murakami $\mathrm{H}$ and Ootao Y. Transient thermal stress analysis of a laminated composite beam. J Therm Stresses 1989; 12: 25-39.

16. Verchery G. Moisture diffusion in polymer matrix composites with cyclic environmental conditions. In: Proceedings of the 5th European Conference on Composites Materials ECCM-5, Bordeaux, France, 7-10 April 1992, pp.505-510.

17. Kelvin L. The reduction of observation of underground temperature. Trans $R$ Soc Edinburgh 1861; 22: 405.

18. Jedidi J, Jacquemin F and Vautrin A. Accelerated hygrothermal cyclical tests for carbon/epoxy laminates. Composites Part A 2006; 37: 636-645. 